\title{
Torque performance study of magnus wind turbine
}

\begin{abstract}
Current wind turbine technology cannot operate in low wind velocity conditions to produce adequate torque for high capacity generators. This Magnus Wind Turbine (MWT) experiment aims to study the torque performance in relationship with the frequency of rotation of the cylinder blade. The experiment started with the design and fabrication of the MWT through computational calculation and simulation. This study solves the relationship gap between the torque generated and the rotation frequency of the cylinder blade by building upon previous research where only one type of rotation frequency of the cylinder blade was used. The research is done inside a wind tunnel by attaching a spring balance to the rotor shaft through a rope. The spring balance stops the rotor shaft from rotating at certain positions and the corresponding torques were measured, while the rotation frequency of the cylinder blade and wind velocity are controlled. The results show a positive trend that the torque generated will increases as rotation frequency of the cylinder blade and wind velocity are increased. Therefore, the results indicate the possibility that a higher torque can be produced by MWT in countries with low wind velocity.
\end{abstract}

Keyword: Magnus wind turbine; Reynold numbers; Torque performance; Wind energy 